\title{
Hace poco y no hace mucho. El efecto en la medición de la migración por cambios en la duración de la residencia
}

\author{
Virgilio Partida-Bush \\ Facultad Latinoamericana de Ciencias Sociales-Sede México
}

\section{Resumen}

Con base en cuatro levantamientos de la Encuesta Nacional de la Dinámica Demográfica (1992, 1997, 2009 y 2014) se comparan los migrantes interestatales según la residencia uno y cinco años antes de la entrevista. Se verifica que el conteo del lustro excede entre tres y cuatro veces al del año. No se alcanza el quíntuple por la migración de retorno, es decir, personas que cinco años antes y al momento de la entrevista vivían en la misma entidad federativa, pero en una entidad federativa distinta un año antes. Aunque se advierte el patrón por edad típico de las tasas de migración para ambos periodos, en las tasas del año previo aparecen irregularidades hacia el final de la vida, lo cual se debe a que un menor tamaño de muestra repercute en estimaciones menos precisas que para el quinquenio anterior, donde la mayor frecuencia del fenómeno conlleva un mayor tamaño de la muestra y, por ende, estimaciones más precisas. Se concluye que en los censos y encuestas por muestreo de gran tamaño, se debe seguir prefiriendo cinco años antes sobre uno, con el fin de maximizar el monto de migrantes.

Palabras clave: Migración interna, periodo de residencia.

\section{Recently and not long ago.}

The effect of changes in the length of residence on the measurement of migration

\section{Abstract}

It uses the 1992, 1997, 2009 and 2014 data from the Dynamic Demographic National Survey (ENADID. It compares interstate migrants by place of residence one and five years before the interview. It verifies that the five years account exceeds three to four times the one year account. The quintuple is not reached because the return migration, i.e., people living in the same state five years before and at the moment of the interview, but residing in a different state one year before. The typical age-pattern of the internal migration rates occurs, but there are some irregularities towards the end of life in the one year estimates, because the sample size for one year is smaller than the lustrum sample. It is concluded that is better five years before than one in large sample surveys and censuses, in order to maximize the amount of migrants.

Key words: Internal migration, length of residence.

* El autor agradece los valiosos comentarios y sugerencias de un dictaminador anónimo.

Recibido: 28 de enero de 2019

Aprobado: 20 de mayo de 2019 


\section{INTRODUCCIÓN}

I rápido crecimiento urbano, derivado de la pujante industrialización de los países en vías de desarrollo, sobre todo en los primeros lustros de la segunda mitad del siglo XX, propició que buena cantidad de los estudios de corte demográfico se dedicaran a examinar la migración interna.

La importancia de la movilidad del campo a la ciudad favoreció el levantamiento de encuestas por muestreo en varias ciudades importantes de América Latina (Elizaga, 1970; Alberts, 1977; Balán, Browning y Jelín, 1977; Muñoz, Oliveria y Stern, 1977; Arroyo, Winnie y Velázquez, 1986), con el fin, entre muchos otros objetivos, de inspeccionar la inserción de los migrantes en la urbe de destino.

Esas encuestas, sin embargo, daban solo una idea parcial del fenómeno, pues se referían exclusivamente a los inmigrantes a la ciudad particular donde se aplicaba el cuestionario, difícilmente se podían replicar en otras áreas urbanas del país por el alto costo financiero de su levantamiento, pues la mayoría incluyeron la historia de vida de varios miembros del hogar, y no era recomendable extender los hallazgos y conclusiones a otras realidades.

Si bien, con limitaciones marcadas en su potencial analítico, respecto de las encuestas de hogares por muestreo, los censos de población eran la fuente adecuada para profundizar en el estudio de la migración interna a lo largo del territorio nacional.

La pregunta tradicional del lugar de nacimiento, en censos de población y encuestas de hogares por muestreo, era insuficiente para medir y entender mejor el cuantioso éxodo rural, con lo cual se bosquejaron e incorporaron preguntas específicas sobre la migración reciente. De manera general, dos fueron los diseños:

1. Preguntar por la residencia en una fecha previa determinada.

2. Preguntar si la persona ha vivido fuera del lugar donde reside al momento de la enumeración, y si la respuesta es afirmativa, se solicita el último lugar donde vivió antes de llegar a la residencia actual, y el tiempo de vivir en ésta última. 
La primera forma es más sencilla, aunque también acota las posibilidades analíticas de la segunda, ya que solo capta los migrantes recientes. No obstante, la segunda opción requiere de varias preguntas que, en el caso de México, implicaron una omisión presumible de los migrantes recientes en los censos de población de 1970 y, sobre todo, de 1980 (Partida, 1993). Otra limitante de la segunda opción es que, conforme pasa el tiempo, los migrantes se vuelven más selectos, en el sentido que ellos han permanecido un periodo amplio y no se sabe algo de aquellos que llegaron en la misma época pero que posteriormente se fueron a vivir a otro lado, y también que otros hayan fallecido (Browning y Feindt, 1969: 188). Así, no es fácil caracterizar a los migrantes por periodo de llegada a su residencia actual.

Fue por esas dificultades intrínsecas a la forma de preguntar y la limitante referida, que el Comité de Diseño Conceptual del Censo de Población de 1990 acordó cambiar el esquema por la residencia en una fecha previa determinada.

Igual que en los censos de población de Estados Unidos de 1960 a 1980 y el de Panamá de 1980, se seleccionaron cinco años como la fecha previa, es decir, marzo de 1985, que abarcaba un lustro exacto de la fecha del censo de 1990 de México (19 de marzo).

Desde entonces, en los censos de población de 1990 a 2010, el conteo de 2005 y la Encuesta Intercensal de 2015, la pregunta incluida en la boleta es la residencia cinco años atrás. En algunos foros, se ha propuesto cambiar por un año antes.

El objetivo de este trabajo es analizar el efecto que, en la medición de la migración interna, tiene una fecha previa sobre otra, es decir, un año (un poco antes) en comparación por cinco (no hace mucho). Para ello echamos mano de cuatro levantamientos de la Encuesta Nacional de la Dinámica Demográfica (ENADID), ${ }^{1}$ donde en el cuestionario de hogar hay sendas preguntas que permiten la comparación. Antes de pasar al análisis propio de las diferencias numéricas, se discute brevemente el concepto de migración y las ventajas y desventajas de un periodo u otro (uno o cinco años atrás) para la contabilidad de los desplazamientos territoriales.

1 De los cinco levantamientos, omitimos el de 2006, pues ahí solo se preguntó por la residencia cinco años atrás. 


\section{EL CONCEPTO DE MIGRACIÓN Y SU CAPTACIÓN EN LOS CENSOS DE POBLACIÓN Y ENCUESTAS DE HOGARES}

Migración proviene del vocablo latino migratus que significa "ir de un lugar a otro, desplazarse, cambiar de región” (Gómez de Silva, 1988). Así la migración alude a un movimiento territorial; sin embargo, no todos los desplazamientos son migraciones, es necesario acotar el concepto para precisar el tipo de traslados al que nos estaremos refiriendo cuando hablamos de migraciones o migrantes.

Generalmente, se acepta que el traslado implica el cambio de residencia habitual - temporal o definitivo-, pero además que "la reanudación de la vida (sea) en un lugar nuevo y distinto" (Elizaga y Macisco, 1975: 8), e incluso que al desplazarse se quede fuera del área de influencia de un pueblo o de una ciudad (Oberai 1989: 14-18).

Para nuestros fines y, por la forma como se captó en la ENADID, consideramos como migrantes a toda aquella persona que reconoció, o un tercero por ella, haber vivido un año o cinco años antes en un estado distinto a la entidad federativa de residencia al momento de la encuesta.

La pregunta de cinco años antes es prácticamente la misma en los cuatro levantamientos que consideramos (1992, 1997, 2009 y 2014): "Hace cinco años, es decir, en (mes) de (año), ${ }^{2}$ ¿En qué estado de la República Mexicana o país vivía (nombre de la persona)?”3

Es claro que la pregunta no se puede hacer a un menor de cinco años de edad, pues un lustro atrás aún no nacía. No obstante, dado que cinco años es el momento más lejano del lustro para los individuos de cinco años o más de edad, el nacimiento lo es para los menores. Así, se toma el lugar de nacimiento para los menores de cinco años de edad. La pregunta es igual: “¿En qué estado... o país nació (nombre de la persona)?"

En los levantamientos de 2009 y 2014 la pregunta fue específica para un año atrás: Hace un año, es decir, en mayo (agosto) de 2008 (2013), ¿En qué estado de la República Mexicana o país vivía (nombre de la persona)?" Nuevamente, el lugar de nacimiento se usa para los menores de un año de edad.

En los levantamientos de 1992 y 1997 se incluyó la segunda forma de preguntar que presentamos en la introducción. Con el fin de hacer comparables los cuatro levantamientos de la ENADID, para 1992 y

2 Junio de 1987, junio de 1992, mayo de 2004 y agosto de 2009, respectivamente.

3 El término "de la República" se incorporó en 1997 y "Mexicana" además en 2009 y 2014. 
1997 asimilamos a un año antes a quienes declararon tener residiendo menos de un año en la entidad federativa de residencia al momento de la entrevista. En el anexo se describe la secuencia de las preguntas en los cuestionarios de ambas encuestas.

El principal objetivo de la ENADID es recabar información específica de las mujeres en edad fértil (15 a 49 ó 54 años) en la vivienda mediante un cuestionario particular: el laborioso y a veces complicado llenado de la historia de embarazos, el uso de anticonceptivos, la salud materno-infantil y la actividad sexual; incluso en la versión de 1997 se incluyeron historias de anticoncepción y de uniones consensuales duraderas.

$\mathrm{Si}$ la entrevistadora ${ }^{4}$ estuvo suficientemente capacitada para levantar el complicado cuestionario de la mujer, podemos presumir que lo estuvo también para recabar las preguntas de migración interestatal reciente, y que la información respectiva que se captó en esos cuatro levantamientos es de muy buena calidad - si no es que excelente- . No negamos que, en algunos casos pueda haber algún sesgo o desconocimiento en algunos conceptos del cuestionario del hogar cuando un tercero declara por todos los miembros del hogar; pero al estar acotada nuestra ventaja temporal al lustro previo a la entrevista, también podemos conjeturar que al ser eventos recientes su conocimiento por un tercero es factible.

Así, suponemos que los datos son de calidad y que el único error es el asociado al diseño muestral.

\section{EL ESQUEMA DE ANÁLISIS PARA LA COMPARACIÓN DE LOS DOS PERIODOS DE RESIDENCIA}

Hace ya varios lustros, Hope Eldridge (1965) propuso un esquema para analizar la migración interna en Estados Unidos en el periodo 1955-1960, con base en el lugar de residencia cinco años antes del censo de 1960. La célebre demógrafa norteamericana consideró solo el caso cuando el estado de residencia en 1955 era distinto al de 1960, e identificó tres tipos de movilidad espacial:

1. Primaria. El estado de nacimiento es igual al de residencia en 1955.

2. Secundaria. El estado de nacimiento es distinto tanto al de residencia en 1955 como al de residencia en 1960.

4 Se seleccionan solo mujeres para el levantamiento de los cuestionarios de hogar y de mujer, por la sensibilidad que representan los temas incluidos en el cuestionario particular de la mujer. 
3. Retorno. El estado de nacimiento es igual al de residencia en 1960.

Aquí se propone una adaptación del esquema de Eldridge, pero interesados en la migración interestatal del año previo al levantamiento de la ENADID correspondiente, es decir, cuando la entidad de residencia un año antes es diferente a aquella al momento de la entrevista:

1. Primaria. El estado de residencia en 1987 (1992, 2004 o 2009) es igual al de residencia en 1991 (1996, 2008 o 2013) y diferente al de residencia al momento de la encuesta.

2. Secundaria. El estado de residencia en 1987 (1992, 2004 o 2009) es distinto al de residencia en 1991 (1996, 2008 o 2013) $\mathrm{y}$ ambos diferentes al de residencia al momento de la encuesta.

3. Retorno. El estado de residencia en 1987 (1992, 2004 o 2009) igual al de residencia al momento de la encuesta y distinto al de residencia en 1991 (1996, 2008 o 2013).

Debido a que Eldridge está interesada solo en la migración de 1955-1960, no considera migración previa a 1955 cuando no hay desplazamiento en el quinquenio anterior al censo. ${ }^{5}$ Pero, esa forma de movilidad espacial es de toral importancia para nuestro análisis:

4. Previa. El estado de residencia en 1987 (1992, 2004 o 2009) es distinto al de residencia en 1991 (1996, 2008 o 2013), pero este último es igual al de residencia al momento de la encuesta.

\section{CAMBIOS EN EL MONTO E INTENSIDAD DE LA MIGRACIÓN POR DISTINTOS PERIODOS DE RESIDENCIA}

En la Tabla 1 se reproducen las contabilidades (ponderadas) de la migración según la residencia un año y un lustro antes de la entrevista. Recuérdese que para nuestros fines, la movilidad territorial de referencia es la del año anterior. Con base en los coeficientes de variación que se reproducen en la Tabla 2 , se tiene que todos los flujos son de buena calidad. ${ }^{6}$ En el cuadro anexo se presentan los errores estándar de las estimaciones.

5 El calificativo primaria implica suponer que no hubo migración antes de 1955: “... dejaron su estado de nacimiento después de 1 de abril 1955" (Eldridge, 1965: 446).

6 Martínez (2017) cita que el INEGI considera que un dato es de buena calidad si el coeficiente de variación está por debajo de 15 por ciento, aceptable si se encuentra entre 15 y 25 por ciento y de baja calidad cuando supera 25 por ciento. Las cuatro celdas de la Tabla 2 que excede 15 por ciento a penas serian aceptables y no de buena calidad. 
Tabla 1: México: Migración según fecha de residencia previa, 1987-2014

\begin{tabular}{|c|c|c|c|c|}
\hline \multirow{2}{*}{$\begin{array}{l}\text { Tipo de migración por fecha } \\
\text { de residencia previa }\end{array}$} & \multicolumn{4}{|c|}{ Año de levantamiento de la encuesta } \\
\hline & 1992 & 1997 & 2009 & 2014 \\
\hline $\begin{array}{l}\text { Residentes en el país* } \\
\text { FLUJOS INTERESTATALES }\end{array}$ & 84436600 & 91869978 & 106043660 & 118416685 \\
\hline Un año antes ${ }^{\dagger}$ & 1147226 & 1313000 & 932370 & 1187426 \\
\hline Cinco años antes $\ddagger$ & 4573259 & 4540310 & 3044980 & 3412273 \\
\hline Diferencia & 3426033 & 3227310 & 2112610 & 2224847 \\
\hline Primaria & 750042 & 763496 & 666557 & 737845 \\
\hline Secundaria & 62989 & 95423 & 79603 & 152121 \\
\hline Retorno & 334195 & 454081 & 186210 & 297460 \\
\hline Previa & 3760228 & 3681391 & 2298820 & 2522307 \\
\hline Previa menos retorno & 3426033 & 3227310 & 2112610 & 2224847 \\
\hline TOTAL & 4907454 & 4994391 & 3231190 & 3709733 \\
\hline PORCENTAJE DEL TOTAL & & & & \\
\hline Un año antes & 23.4 & 26.3 & 28.9 & 32.0 \\
\hline Cinco años antes & 93.2 & 90.9 & 94.2 & 92.0 \\
\hline Diferencia & 69.8 & 64.6 & 65.4 & 60.0 \\
\hline Primaria & 15.3 & 15.3 & 20.6 & 19.9 \\
\hline Secundaria & 1.3 & 1.9 & 2.5 & 4.1 \\
\hline Retorno & 6.8 & 9.1 & 5.8 & 8.0 \\
\hline Previa & 76.6 & 73.7 & 71.1 & 68.0 \\
\hline Previa menos retorno & 69.8 & 64.6 & 65.4 & 60.0 \\
\hline TOTAL & 100.0 & 100.0 & 100.0 & 100.0 \\
\hline Cinco años / un año antes & 4.0 & 3.5 & 3.3 & 3.1 \\
\hline Retorno / un año (\%) & 29.1 & 34.6 & 20.0 & 4.9 \\
\hline Previa / cinco años (\%) & 82.2 & 81.1 & 75.5 & 1.2 \\
\hline
\end{tabular}

* Personas cuya residencia un año y un lustro antes era algún Estado de México; es decir, se excluyen aquellos que uno o cinco años antes vivían en otro país.

† Suma de primaria, secundaria y retorno.

‡ Suma de primaria, secundaria y previa.

Fuente: Microdatos de la Encuesta Nacional de la Dinámica Demográfica 1992, 1997, 2009 y 2014.

De acuerdo a la clasificación que hicimos de los migrantes, el monto del año previo es la suma de primaria, secundaria y retorno; mientras para el lustro precedente es primaria, secundaria y previa. ${ }^{7}$ Es claro que la diferencia entre los montos se origina en la migración de retorno y la previa.

En el antepenúltimo renglón de la Tabla 1, se advierte que el monto del lustro no es cinco veces el del año, lo cual es evidente porque, dada la mayor longitud del intervalo temporal, la migración previa debe ser mayor que la de retorno. No obstante, el exceso de cinco años

7 Los retornos se excluyen de la contabilidad de cinco años, pues bajo esa temporalidad no son migrantes, porque un lustro atrás vivían en la misma entidad federativa que al momento de la entrevista, y bajo esa pregunta desconocemos la residencia un año antes. 
sobre uno se redujo con el tiempo; esto se debe a la contracción del distanciamiento entre las migraciones previa y de retorno. Y, aunque la diferencia no es despreciable en términos absolutos, se ha reducido proporcionalmente - respecto del total de migrantes - con el paso de los años.

Tabla 2: México: Coeficientes de variación de la migración según fecha de residencia previa, 1987-2014 (Por cien)

\begin{tabular}{lrrrr}
\hline Tipo de migración por fecha & \multicolumn{5}{c}{ Año de levantamiento de la encuesta } \\
\cline { 2 - 6 } de residencia previa & \multicolumn{1}{c}{1992} & \multicolumn{1}{c}{1997} & 2009 & 2014 \\
\hline Residentes en el país & 1.98 & 0.54 & 0.55 & 0.40 \\
FLUJOS INTERESTATALES & & & & \\
Un año antes & 7.27 & 3.46 & 5.64 & 3.52 \\
Cinco años antes & 5.79 & 2.33 & 3.05 & 2.38 \\
Diferencia & 6.84 & 3.04 & 3.47 & 3.14 \\
Primaria & 8.91 & 4.83 & 7.01 & 4.32 \\
Secundaria & 16.71 & 11.27 & 15.97 & 8.39 \\
Retorno & 7.99 & 4.96 & 7.92 & 5.66 \\
Previa & 6.24 & 2.61 & 3.18 & 2.69 \\
Previa menos retorno & 6.84 & 3.04 & 3.47 & 3.14 \\
TOTAL & 5.51 & 2.18 & 2.95 & 2.26 \\
PORCENTAJE DEL TOTAL & & & & \\
Un año antes & 6.22 & 3.10 & 4.34 & 2.83 \\
Cinco años antes & 0.62 & 0.49 & 0.47 & 0.48 \\
Diferencia & 2.70 & 1.71 & 2.16 & 1.96 \\
Primaria & 7.61 & 4.46 & 5.72 & 3.66 \\
Secundaria & 15.41 & 11.14 & 15.71 & 8.03 \\
Retorno & 8.45 & 4.92 & 7.76 & 5.53 \\
Previa & 1.90 & 1.10 & 1.76 & 1.33 \\
Previa menos retorno & 2.70 & 1.71 & 2.16 & 1.96 \\
Cinco años / un año antes & 6.65 & 3.36 & 4.46 & 3.08 \\
Retorno / un año (\%) & 6.34 & 4.33 & 8.08 & 4.93 \\
Previa / cinco años (\%) & 1.56 & 0.96 & 1.73 & 1.19 \\
\hline
\end{tabular}

Fuente: Microdatos de la Encuesta Nacional de la Dinámica Demográfica 1992, 1997, 2009 y 2014.

Si bien el monto de migrantes aumenta conforme se alarga el periodo de la residencia, la intensidad del fenómeno (tasas medias anuales) es mayor para la movilidad anual que la quinquenal, y cada vez más distante, como se advierte en la Tabla 3; incluso, supera casi tres cuartas partes en el periodo 2009-2014. ${ }^{8}$ Los coeficientes de

8 Las tasas se calcularon al dividir los migrantes entre la población residente (primer renglón de la Tabla 1). Las de cinco años antes se dividieron entre cinco para anualizarlas y hacerlas comparables con las tasas del año anterior. 
variación, en la última columna, muestran que las estimaciones son de buena calidad, salvo la diferencia entre tasas de 1987-1992 que serían estimaciones de baja calidad (coeficiente de variación de 31.2 por ciento).

Tabla 3: México: Tasas de migración según fecha de residencia previa, 1987-2014 (Tasas por mil)

\begin{tabular}{|c|c|c|c|c|c|}
\hline & \multirow[b]{2}{*}{ Tasa } & \multirow{2}{*}{$\begin{array}{l}\text { Error } \\
\text { estándar }\end{array}$} & $\begin{array}{l}\text { Límites } \\
\text { confianza }\end{array}$ & $90 \%$ & \multirow{2}{*}{$\begin{array}{l}\text { Coeficiente } \\
\text { de variación } \\
(\%)\end{array}$} \\
\hline & & & Inferior & Superior & \\
\hline \multicolumn{6}{|l|}{ 1987-1992 } \\
\hline Un año antes & 13.6 & 0.91 & 12.1 & 15.1 & 6.73 \\
\hline Cinco años antes & 10.8 & 0.56 & 9.9 & 11.8 & 5.16 \\
\hline Un año menos cinco años & 2.8 & 0.86 & 1.3 & 4.2 & 31.24 \\
\hline $\begin{array}{l}\text { Porcentaje de exceso } \\
1992-1997\end{array}$ & 25.4 & 8.34 & 11.7 & 39.2 & 6.65 \\
\hline Un año antes & 14.3 & 0.50 & 13.3 & 15.3 & 3.48 \\
\hline Cinco años antes & 9.9 & 0.23 & 9.4 & 10.3 & 2.34 \\
\hline Un año menos cinco años & 4.4 & 0.46 & 3.5 & 5.3 & 10.45 \\
\hline \multicolumn{6}{|l|}{ 2004-2009 } \\
\hline Un año antes & 8.8 & 0.49 & 8.0 & 9.6 & 5.59 \\
\hline Cinco años antes & 5.7 & 0.17 & 5.5 & 6.0 & 3.02 \\
\hline Un año menos cinco años & 3.0 & 0.41 & 2.4 & 3.7 & 13.45 \\
\hline Porcentaje de exceso & 53.1 & 6.83 & 41.9 & 64.3 & 4.46 \\
\hline \multicolumn{6}{|l|}{ 2004-2009 } \\
\hline Un año antes & 10.0 & 0.35 & 9.3 & 10.7 & 3.53 \\
\hline Cinco años antes & 5.8 & 0.14 & 5.5 & 6.0 & 2.40 \\
\hline Un año menos cinco años & 4.3 & 0.31 & 3.7 & 4.9 & 7.20 \\
\hline Porcentaje de exceso & 74.0 & 2.28 & 63.5 & 84.5 & 3.08 \\
\hline
\end{tabular}

Fuente: Microdatos de la Encuesta Nacional de la Dinámica Demográfica 1992, 1997, 2009 y 2014.

La tendencia descendente en las tasas de migración interestatal total data de los años ochenta del siglo pasado (Partida, 2014).

En la Tabla 4 y en la Figura 1 se reproducen las tasas específicas por edad para las dos últimas ENADID. Se aprecia la pauta etaria general de la migración interna (Rogers y Castro, 1981), aunque con algunas irregularidades hacia el final de la vida en las tasas del año previo, debido al bajo tamaño de muestra para un fenómeno estadísticamente escaso como es la migración. 
Tabla 4: México: Tasas de migración según fecha de residencia previa, 2004-2014 (Tasas por mil)

\begin{tabular}{|c|c|c|c|c|c|c|c|c|c|c|}
\hline \multirow[b]{3}{*}{ Edad } & \multicolumn{5}{|c|}{ Un año antes } & \multicolumn{5}{|c|}{ Cinco años antes } \\
\hline & \multirow[b]{2}{*}{ Tasa } & \multirow{2}{*}{$\begin{array}{l}\text { Error } \\
\text { estándar }\end{array}$} & \multicolumn{2}{|c|}{ Límites 90\% } & \multirow{2}{*}{$\begin{array}{l}\text { Coef. de } \\
\text { var. (\%) }\end{array}$} & \multirow[b]{2}{*}{ Tasa } & \multirow{2}{*}{$\begin{array}{l}\text { Error } \\
\text { estándar }\end{array}$} & \multicolumn{2}{|c|}{ Límites 90\% } & \multirow{2}{*}{$\begin{array}{l}\text { Coef. de } \\
\text { var. (\%) }\end{array}$} \\
\hline & & & Inferior & Superior & & & & Inferior & Superior & \\
\hline \multicolumn{11}{|c|}{ 2004-2009 } \\
\hline $0-4$ & 15.5 & 1.4 & 13.4 & 17.9 & 8.9 & 9.9 & 0.6 & 9.0 & 10.8 & 5.6 \\
\hline $5-9$ & 9.1 & 1.2 & 7.4 & 11.3 & 12.9 & 5.7 & 0.4 & 5.1 & 6.3 & 6.5 \\
\hline $10-14$ & 5.2 & 0.9 & 3.9 & 7.0 & 17.4 & 4.2 & 0.3 & 3.7 & 4.7 & 7.2 \\
\hline $15-19$ & 9.1 & 0.7 & 8.0 & 10.3 & 7.5 & 6.0 & 0.3 & 5.4 & 6.5 & 5.7 \\
\hline $20-24$ & 13.9 & 1.1 & 12.2 & 15.7 & 7.6 & 8.6 & 0.4 & 7.9 & 9.3 & 4.9 \\
\hline $25-29$ & 15.1 & 1.3 & 13.2 & 17.4 & 8.4 & 8.8 & 0.4 & 8.1 & 9.5 & 5.1 \\
\hline $30-34$ & 12.8 & 2.2 & 9.7 & 16.9 & 16.8 & 7.9 & 0.7 & 6.8 & 9.1 & 8.6 \\
\hline $35-39$ & 8.3 & 0.9 & 6.9 & 9.8 & 10.6 & 6.1 & 0.4 & 5.5 & 6.8 & 6.7 \\
\hline $40-44$ & 6.5 & 1.1 & 5.0 & 8.7 & 17.0 & 4.4 & 0.3 & 3.9 & 5.0 & 7.8 \\
\hline $45-49$ & 4.4 & 0.8 & 3.3 & 5.9 & 17.0 & 3.3 & 0.3 & 2.9 & 3.8 & 7.9 \\
\hline $50-54$ & 4.3 & 1.2 & 2.7 & 6.8 & 28.3 & 3.1 & 0.4 & 2.5 & 3.8 & 12.6 \\
\hline $55-59$ & 2.6 & 0.5 & 1.9 & 3.7 & 19.6 & 2.0 & 0.2 & 1.6 & 2.4 & 12.0 \\
\hline $60-64$ & 2.1 & 0.5 & 1.4 & 3.0 & 23.2 & 2.3 & 0.3 & 1.8 & 2.9 & 14.1 \\
\hline $65-69$ & 3.4 & 1.0 & 2.1 & 5.5 & 28.3 & 2.0 & 0.3 & 1.6 & 2.5 & 14.3 \\
\hline $70-74$ & 2.7 & 0.9 & 1.6 & 4.8 & 34.3 & 2.1 & 0.5 & 1.4 & 3.1 & 23.8 \\
\hline $75-79$ & 3.1 & 2.0 & 1.0 & 9.0 & 66.2 & 2.3 & 0.5 & 1.5 & 3.4 & 23.8 \\
\hline $80-84$ & 7.0 & 4.3 & 2.6 & 19.0 & 60.6 & 3.0 & 1.0 & 1.8 & 5.1 & 32.6 \\
\hline $85-89$ & 3.8 & 1.9 & 1.6 & 8.8 & 51.4 & 1.2 & 0.5 & 0.6 & 2.2 & 38.0 \\
\hline \multicolumn{11}{|c|}{ 2009-2014 } \\
\hline $0-4$ & 16.5 & 1.1 & 14.8 & 18.3 & 6.6 & 11.0 & 0.5 & 10.2 & 11.8 & 4.3 \\
\hline $5-9$ & 7.4 & 0.6 & 6.5 & 8.5 & 8.2 & 5.5 & 0.3 & 5.0 & 6.0 & 5.2 \\
\hline $10-14$ & 6.1 & 0.6 & 5.2 & 7.3 & 10.4 & 4.4 & 0.3 & 4.0 & 4.8 & 5.9 \\
\hline $15-19$ & 11.8 & 0.8 & 10.5 & 13.3 & 7.0 & 5.5 & 0.3 & 5.1 & 6.0 & 5.0 \\
\hline $20-24$ & 16.8 & 1.0 & 15.2 & 18.6 & 6.1 & 8.2 & 0.4 & 7.6 & 8.8 & 4.3 \\
\hline $25-29$ & 14.7 & 1.0 & 13.2 & 16.5 & 6.8 & 8.4 & 0.4 & 7.8 & 9.1 & 4.5 \\
\hline $30-34$ & 13.8 & 1.1 & 12.1 & 15.6 & 7.7 & 8.1 & 0.4 & 7.5 & 8.8 & 4.8 \\
\hline $35-39$ & 11.6 & 1.1 & 9.9 & 13.5 & 9.5 & 6.8 & 0.4 & 6.2 & 7.4 & 5.3 \\
\hline $40-44$ & 8.4 & 0.9 & 7.0 & 10.0 & 10.7 & 4.7 & 0.3 & 4.2 & 5.2 & 6.1 \\
\hline $45-49$ & 5.5 & 0.8 & 4.4 & 6.9 & 13.9 & 3.3 & 0.2 & 2.9 & 3.7 & 7.3 \\
\hline $50-54$ & 6.5 & 0.8 & 5.2 & 8.0 & 13.0 & 3.1 & 0.3 & 2.7 & 3.6 & 8.3 \\
\hline 55-59 & 6.3 & 1.2 & 4.6 & 8.5 & 18.8 & 2.8 & 0.3 & 2.4 & 3.3 & 10.7 \\
\hline $60-64$ & 4.0 & 0.8 & 2.9 & 5.4 & 18.9 & 2.4 & 0.2 & 2.0 & 2.8 & 10.5 \\
\hline $65-69$ & 4.3 & 1.0 & 2.9 & 6.4 & 24.1 & 2.0 & 0.3 & 1.6 & 2.5 & 13.8 \\
\hline $70-74$ & 5.4 & 1.3 & 3.6 & 8.0 & 23.8 & 1.4 & 0.3 & 1.0 & 1.9 & 18.7 \\
\hline $75-79$ & 2.5 & 0.7 & 1.6 & 3.9 & 28.2 & 1.9 & 0.4 & 1.3 & 2.7 & 22.1 \\
\hline $80-84$ & 3.1 & 1.0 & 1.8 & 5.3 & 33.1 & 2.9 & 0.5 & 2.2 & 3.8 & 17.2 \\
\hline 85-89 & 3.5 & 1.4 & 1.8 & 6.7 & 39.8 & 1.6 & 0.4 & 1.1 & 2.5 & 25.1 \\
\hline
\end{tabular}

Fuente: Microdatos de la Encuesta Nacional de la Dinámica Demográfica 1992, 1997, 2009 y 2014.

En la última columna de los paneles correspondientes a cada lustro y duración de la residencia en la Tabla 4, se advierte que las estimaciones se vuelven menos precisas conforme avanza la edad, sobre todo a partir de 50 años de edad.

Debido a la mayor cuantía de migrantes durante el quinquenio previo que para el año anterior de ambos levantamientos de la 
encuesta, las estimaciones de cinco años antes son más precisas que las del año precedente, lo cual se puede ver también en la Figura 1, donde la pauta es más suave para un lapso de cinco años que para un año, y mientras se identifica claramente el llamado pico del retiro (la campana entre 70 y 85 años de edad) en la migración del lustro, el patrón vaciante del año previo no permite visualizarlo de manera tan evidente.

Figura 1: México: Tasas de migración por periodo de residencia, 2004-2014

Por mil habitantes

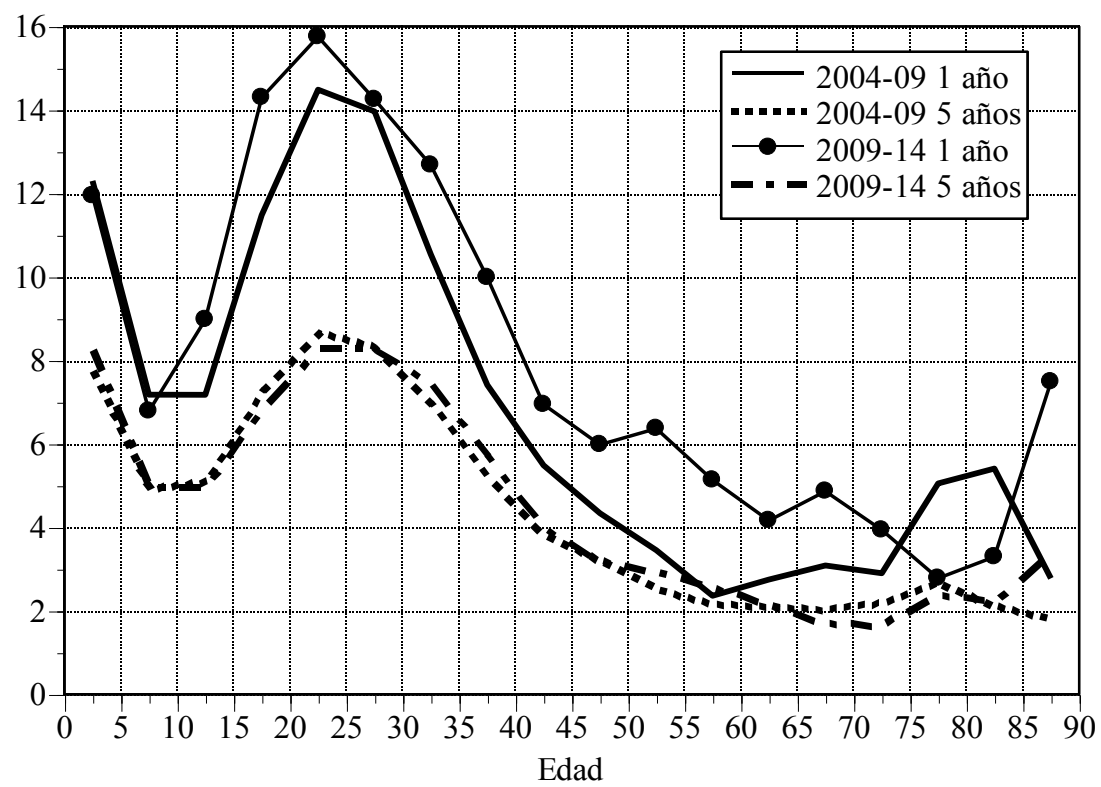

Fuente: Tabla 4.

\section{Conclusiones}

La alta movilidad del campo a la ciudad, en las primeras décadas de la segunda mitad del siglo pasado, favoreció el diseño e incorporaron de preguntas específicas sobre la migración reciente en los censos de población. En los recuentos de habitantes de México de 1960, 1970 y 1980 se incluyeron preguntas donde se indagaba si la persona había vivido en una entidad federativa distinta a donde residía al momento de la enumeración, y si la respuesta era afirmativa, se solicitaba el 
último lugar donde vivió antes de llegar a la residencia actual, y el tiempo de vivir en ésta última.

La complejidad de las preguntas fue tal, que originó una omisión presumible de los migrantes recientes en los censos de población de 1970 y, sobre todo, de 1980 (Partida, 1993). Ante tal situación, el Comité de Diseño Conceptual del Censo de Población de 1990 optó por cambiar por una pregunta más simple: solicitar el estado de residencia cinco años antes. Este esquema se ha mantenido en los censos posteriores, el conteo de 2005, la Encuesta Intercensal de 2015 y otras encuestas por muestreo.

En distintos foros se ha cuestionado si cinco años es la mejor duración para captar las migraciones en lugar de un año. En este trabajo, con base en cuatro levantamientos de la Encuesta Nacional de la Dinámica Demográfica (ENADID), donde se ha incluido la residencia uno y cinco años antes, hacemos una somera comparación de flujos y tasas para ambos periodos de la residencia anterior.

La contabilidad de migrantes para el lustro previo es, obviamente, mayor que la del año anterior, pero, en ninguno de los quinquenios revisados, el monto del lustro es cinco veces el del año, como pudiera esperarse; incluso, la proporción desciende de manera monótona de 4.0 veces a 3.1 veces al cabo del tiempo.

En las tasas medias anuales, el escenario es diametralmente opuesto: en los cuatro periodos la tasa del año anterior supera a la del quinquenio, aumentando continuamente el excedente proporcional de 25 a 74 por ciento.

En el ámbito de la Demografía, si bien los montos son importantes, las tasas también lo son, y muchas veces las segundas describen mejor la historia del fenómeno que los primeros, bajo la premisa que los volúmenes de migrantes dependen estrechamente del número de residentes.

Bajo esta línea de razonamiento, se debiera preferir la migración del año previo al levantamiento de una encuesta censal o por muestreo, considerando además que la tendencia de los procesos demográficos es pausada, y que la tasa del año previo debe ser similar a la del quinquenio, si en el lustro se contabilizaran a todos los desplazamientos y no solo la resta que hemos denominado migración "previa" (aquellos que se desplazan entre cinco y un año antes de la entrevista). 
Al comparar las tasas específicas por edad, la pauta del periodo quinquenal exhibe un patrón más suave que el patrón del intervalo anual; $y$, dada la mayor densidad de migrantes del lustro que del año, las estimaciones son más precisas, sobre todo en la adultez madura y tardía.

En encuestas por muestreo, que no son representativas de toda la movilidad interestatal, como es el caso de la ENADID, se pueden incluir ambas preguntas: la residencia un año y cinco años antes; no obstante, en los ejercicios censales o encuestas de gran tamaño, como la intercensal de 2015, es conveniente elegir solo uno de ambos periodos, dada la necesidad de incorporar otras variables de igual o mayor importancia en el cuestionario.

En aras de maximizar el volumen de los flujos migratorios, a la luz del alto porcentaje de desplazamientos que se recuperan al considerar la residencia cinco años antes $(60$ a 70 por ciento en la Tabla 1) y considerando que un lustro es suficientemente corto para que la ocurrencia de un número importante de cambios de residencia múltiples (a lo más 12.1 por ciento combinado de secundaria y retorno en la Tabla 1) subestimara el monto e intensidad de la migración, ${ }^{9}$ en los censos y encuestas por muestreo de gran tamaño, se debe seguir prefiriendo cinco años antes que uno.

Después de todo, no hace mucho no es tan distinto de hace poco.

9 Porque en el caso quinquenal, al asimilar migraciones a migrantes, se supone implícitamente que durante el lustro hay un solo cambio de residencia o ninguno; supuesto totalmente adecuado en una duración anual. 


\section{ANEXo}

La secuencia de preguntas en 1992 fue:

a) Antes de vivir en el estado de (NOMBRE DEL ESTADo) ¿(NOMBRE) vivió en otro estado o país aunque haya sido por poco tiempo?

b) ¿Cuánto tiempo hace que vive (NOMBRE) en el estado de (NOMBRE DEL ESTADO) (desde que llegó la última vez? MENOS DE UN MES; NO SABE; AÑOS; MESES.

c) Si la respuesta en b) es siempre pregunte: entonces (NOMBRE) tiene (EDAD) años (meses) viviendo aquí. ¿Esto es correcto? SI No HA VIVIDO EN OTRO ESTADO O PAÍS, PASE A (pregunta de residencia anterior de municipio y localidad similar a estado o país) y después a residencia cinco años antes.

Si ha vivido en otro estado o país, también se le pregunta por residencia anterior de municipio y localidad.

La secuencia de preguntas en 1997 fue:

a) ¿(NOMBRE) ha vivido en otro estado o país aunque haya sido por poco tiempo? Si la respuesta es afirmativa se le pregunta por la residencia anterior y años y meses de vivir en la residencia al momento de la entrevista.

b) Si contesta que no en a) se le pregunta: entonces (NOMBRE) tiene (EDAD) años viviendo en (ENTIDAD DE LA ENTREVISTA) Si la respuesta es afirmativa, se le pregunta por residencia anterior de municipio y localidad. Si la respuesta es negativa se le pregunta por estado o país de residencia anterior.

Mientras en la encuesta de 1992 la pregunta de residencia cinco años antes fue después de la batería de preguntas de residencia anterior, en el levantamiento de 1997 la precedió. 
Tabla A: México: Errores estándar de la migración según fecha de residencia previa, 1987-2014

\begin{tabular}{lrrrr}
\hline \multirow{2}{*}{$\begin{array}{l}\text { Tipo de migración por } \\
\text { de residencia previa }\end{array}$} & \multicolumn{4}{c}{ fecha de levantamiento de la encuesta } \\
\cline { 2 - 5 } Residentes en el país & 1992 & \multicolumn{1}{c}{1997} & 2009 & \multicolumn{1}{c}{ Añ4 } \\
\hline FLUJOS INTERESTATALES & 1675113 & 497458 & 581523 & 473928 \\
Un año antes & 83432 & 45442 & 52567 & 41855 \\
Cinco años antes & 264960 & 105733 & 92922 & 81269 \\
Diferencia & 234500 & 98080 & 73370 & 69818 \\
Primaria & 66859 & 36886 & 46723 & 31859 \\
Secundaria & 10526 & 10758 & 12709 & 12762 \\
Retorno & 26696 & 22536 & 14747 & 16831 \\
Previa & 234704 & 96050 & 72998 & 67883 \\
Previa menos retorno & 234500 & 98080 & 73370 & 69818 \\
Total & 270612 & 108853 & 95332 & 83964 \\
PORCENTAJE DEL TOTAL & & & & \\
Un año antes & 1.454 & 0.814 & 1.252 & 0.907 \\
Cinco años antes & 0.575 & 0.447 & 0.447 & 0.443 \\
Diferencia & 1.886 & 1.105 & 1.414 & 1.177 \\
Primaria & 1.163 & 0.681 & 1.179 & 0.728 \\
Secundaria & 0.198 & 0.213 & 0.387 & 0.329 \\
Retorno & 0.575 & 0.447 & 0.447 & 0.443 \\
Previa & 1.454 & 0.814 & 1.252 & 0.907 \\
Previa menos retorno & 1.886 & 1.105 & 1.414 & 1.177 \\
Cinco años / un año antes & 0.265 & 0.116 & 0.146 & 0.089 \\
Retorno / un año (\%) & 1.848 & 1.498 & 1.613 & 1.235 \\
Previa / cinco años (\%) & 1.281 & 0.780 & 1.303 & 0.882 \\
\hline
\end{tabular}

Fuente: Microdatos de la Encuesta Nacional de la Dinámica Demográfica 1992, 1997, 2009 y 2014.

\section{REFERENCIAS BIBLIOGRÁFICAS}

Alberts, Joop. (1977). Migración hacia áreas metropolitanas de América Latina. Un estudio comparativo. Santiago de Chile: Centro Latinoamericano de Demografía.

Arroyo, Jesús, William Winnie y Luis A. Velázquez. (1986). Migración a centros urbanos de una región de fuerte emigración. El caso del occidente de México. Guadalajara: Centro de Investigaciones Sociales y Económicas de la Facultad de Economía de la Universidad de Guadalajara.

Balán Jorge, Harley Browning y Elizabeth Jelín. (1977). El hombre en una sociedad en desarrollo. México: Fondo de Cultura Económica.

Browning, Harley L. y Waltraur Feindt. (1969). "Selectividad de migrantes a una metrópoli en un país en desarrollo: estudio de un caso mexicano". Demografía y Economía, 3(2). No. 8, pp. 186-200.

Eldridge, Hope T. (1965). "Primary, Secondary, and Return Migration in the United States, 1955-60". Demography, 2, pp. 444-455. 
Elizaga, Juan C. (1970). Migraciones a las áreas metropolitanas de América Latina. Santiago de Chile: Centro Latinoamericano de Demografía.

Elizaga, Juan C. y John J. Macisco Jr. (1975). Migraciones internas. Teoría, método y factores sociológicos. Santiago de Chile: Centro Latinoamericano de Demografía.

Gómez de Silva, Guido. (1988). Breve diccionario etimológico de la lengua española. , México: El Colegio de México y Fondo de Cultura Económica.

Martínez, Julio C. (2017).“Una aproximación metodológica al uso de datos de encuestas en hogares". Realidad, Datos y Espacio, 8(2), pp. 53-71.

Muñoz, Humberto, Orlandina de Oliveria y Claudio Stern. (1977). Migración y desigualdad social en la Ciudad de México. México: Instituto de Investigaciones Sociales de la Universidad Nacional Autónoma de México y El Colegio de México.

Oberai, Amarjit S. (1989). Migración, urbanización y desarrollo. Ginebra: Oficina Internacional de Trabajo. (Estudios básicos para la formación en población, recursos humanos y planificación del desarrollo 5)

Partida, Virgilio. (1993). Estimación de los niveles de la migración en el censo de México de 1980. México: Centro de Estudios en Población y Salud, Secretaría de Salud.

Partida, Virgilio. (2014). "De los desplazamientos del campo a la ciudad a los traslados interurbanos". En Cecilia Rabell (coord.), Los Mexicanos. Un balance del cambio demográfico. Fondo de Cultura Económica, México, pp. 389-444.

Rogers, Andrei y Luis J. Castro. (1981). Model Migration Schedules. International Institute for Applied Systems Analysis (IIASA), Laxenburg, Austria. (RR-81-30).

\section{RESUMEN CURRICULAR DEL AUTOR}

Virgilio Partida Bush

Actuario y Doctor en Ciencias Políticas y Sociales con orientación en Sociología por la Universidad Nacional Autónoma de México y Maestro en Demografía por El Colegio de México. Ha sido profesor-investigador en el CEDUA de El Colegio de México y Director de Investigación Demográfica y Director General de Estudios Sociodemográficos y Prospectiva en el Consejo Nacional de Población y actualmente es profesor e investigador en la Facultad Latinoamericana de Ciencias Sociales (FLACSO)-Sede México. Es autor o coautor de 232 libros y más de cien artículos, capítulos en libros y colaboraciones periodísticas. Es miembro del Sistema Nacional de Investigadores nivel III y Profesor Honorífico del CIESS.

Dirección electrónica: vpartida@prodigy.net.mx 